\title{
Determination of Metastatic Axillary Lymph Node in Breast Cancer: Differentiation with Dynamic MRI Examination by Signal Intensity-Time Curves
}

\author{
—Diagnosis of Metastatic Axillary Lymph Nodes on Dynamic MRI
}

\author{
Duzgun Yildirim', Baki Ekci², Bengi Gurses ${ }^{3}$, Ahmet Kaur ${ }^{4}$ \\ ${ }^{1}$ Kasimpasa Military Hospital, Department of Radiology, İstanbul, Turkey; ${ }^{2}$ Yeditepe University Hospital, Department of Surgery, \\ İstanbul, Turkey; ${ }^{3}$ Yeditepe University Hospital, Department of Radiology, İstanbul, Turkey; ${ }^{4}$ E-sitopatoloji Laboratories, Depart- \\ ment of Cytohistopathology, İstanbul, Turkey. \\ Email: \{yildirimduzgun, bengur0,drbaki\}@yahoo.com, esitopatoloji@gmail.com
}

Received June $18^{\text {th }}, 2011$; revised July $20^{\text {th }}, 2011$; accepted July $31^{\text {st }}, 2011$.

\begin{abstract}
Purpose: We aimed to evaluate the role of dynamic magnetic resonance imaging (MRI) in the detection of axillary lymph node metastasis based on the signal intensity-time curves. Materials and Methods: The data of 120 patients (benign patients, $n=91$; malignant patients, $n=29)$ who underwent dynamic breast MRI were reviewed. The lymph nodes with the strongest criteria for malignancy (morphological-dynamic properties) were included in the analysis. Signal intensity-time curves were plotted by the software. Results: Of 29 patients with breast cancer, axillary lymph nodes were involved in 21 and not involved in the remaining 8. There was no significant difference between benign and malignant cases in terms of the distributions of Type Ia, Type Ib and Type IV curves ( $p=0.12)$, whereas a significant difference was found between benign and malignant cases in terms of the distributions of Type II and III curves $(p<0.01)$. Benign lymph nodes dominantly displayed Type III curves (66.2\%), whereas malignant ones dominantly displayed Type II curves (57.2\%). Conclusion: On dynamic MRI studies, benign and metastatic lymph nodes display different signal intensity-time curves.
\end{abstract}

Keywords: Dynamic Magnetic Resonance Imaging, Breast Neoplasms, Axillary Lymph Nodes, Lymphatic Metastasis, Sensitivity-Specificity

\section{Introduction}

Carcinoma of the breast is the second most common malignancy in females, with an increasing incidence of approximately $3 \%$ per year $[1,2]$. In addition to tumor size and histological type, axillary lymph node involvement is also an important prognostic factor in breast cancer. Once the diagnosis of breast cancer is established, almost all patients with invasive and many patients with noninvasive cancer undergo a surgical procedure for the pathologic evaluation of their axillary lymph nodes. Axillary lymph node involvement is the most important prognostic factor in determining survival of patients with breast cancer [3]. Axillary dissection is a separate procedure requiring hospitalization, general anesthesia, and a 1- to 2-week period of postoperative drain care $[3,4]$. The effect of axillary lymph node dissection on long-term treatment results in invasive breast cancer is controversial; it is in fact frequently questioned because it is associated with high morbidity rates and neoadjuvant chemotherapy is already administered in aggressive local cancers [5]. Except for these certain situations, however, there are also studies suggesting that it is essential to detect axillary lymph node involvement in patients with breast cancer; since, it is a very important prognostic indicator in these patients and radiotherapy is indicated if the patient had $>4$ positive nodes $[2,6]$. Several studies have reported that physical examination, mammography, ultrasonography (US) and even other imaging methods performed via various radionuclides may not be sufficient in the detec- 
tion of axillary lymph node involvement [1,7]. Up to date, although there is extensive data regarding primary tumor in numerous dynamic contrast-enhanced breast MRI (DCE-MRI) studies, there is less information about the axillary region $[8,9]$. In the present study, we aimed to determine metastatic axillary lymph node involvement by a simple and noninvasive method with focusing especially on the dynamic signal-intensity curve characteristics of the nodes.

\section{Materials and Methods}

\subsection{Patients}

A total of 120 patients (mean age, $43 \pm 7.2$ years; age range, 24 - 61 years; 91 benign patients and 29 malignant patients) who underwent breast and axillary surgery (for malignant lesions) and DCE-MRI preoperatively between January 2008 and December 2009 in our center were included in the present study. Medical records of these patients, including images, as well as US/mammography findings and clinical characteristics were achieved from the hospital records. This retrospective study was approved by the institutional ethical board. Patients with contradictory medical records, those without histopathological diagnoses or those without preoperative dynamic breast MRI evaluations were excluded $(n=41)$.

\subsection{Magnetic Resonance Imaging Protocol and Related Dynamic Data Processing}

Magnetic resonance imaging was performed on a 1.5 Tesla system (Siemens Symphony, Erlangen, Germany) with the patient in the prone position and the breast suspended in a dedicated large breast coil. Another inclusion criterion of the study was the appearance of axilla in the image field. After obtaining an axial localizer sequence, axial T1-weighted images [turbo-spin echo (TSE); repetition time (TR), $613 \mathrm{msec}$; echo time (TE), $9.1 \mathrm{msec}$; section thickness (ST), $3.5 \mathrm{~mm}$, axial short $\mathrm{T} 1$ inversion recovery (STIR) sequences (TE: $9370 \mathrm{msec}$, TE: $70 \mathrm{msec}$, ST: $3.5 \mathrm{~mm}$ ) were acquired [field of view (FOV): $400 \times$ $350 \mathrm{~mm}]$. On dynamic evaluation, a standard dose $(0.1$ $\mathrm{mmol} / \mathrm{kg}$ ) of contrast agent with gadodiamide was injected through antecubital vein in each patient immediately following the acquisition of precontrast series. Following the injection of contrast agent, $15 \mathrm{~mL}$ physiological saline flush was injected in each patient. After the contrast injection, DCE-MRI was performed with gradient-recalled sequences (TR, $4.4 \mathrm{msec}$; TE, $1.6 \mathrm{msec}$; flip angle, 12; FOV , $350 \times 350 \mathrm{~mm}, 256 \times 128$; section thickness $1.5 \mathrm{~mm}$ with no section gap). Including those acquired during the precontrast period, a total of 7 series each consisting of 80 consecutive images were acquired in every 51 seconds. Six subtracted images (image series achieved by subtracting the precontrast image from each series) were transferred to the mean curve analysis (Siemens medical solutions, Erlangen, Germany) program in the work station. Contrast dynamic curves were plotted from the cortices of axillary lymph nodes (separately from upper, mid and lower poles) exhibiting corticomedullary contrast enhancement by placing a round-shaped ROI with $1.0 \mathrm{~mm}$ in diameter. After the dynamic study, sagittal late postcontrast fat-saturated T1-weighted TSE images (TR, $532 \mathrm{msec}$; TE, $4.01 \mathrm{msec}$; ST, $3.0 \mathrm{~mm}$; FOV: $400 \times 400 \mathrm{~mm}$ ) were acquired in order to recheck and correlate the lesions and axillary lymph nodes.

Biopsy was performed in dominant lymph node or nodes visualized in axillary images in all cases. The dominant lymph nodes were identified according to the following criteria: asymmetric cortical thickening, cortical irregularity, abnormal growth, cortical heterogeneity, hilar deformation and loss of fatty sinus, loss of ovality (rounding). Regions of interests (ROIs), $1.0 \mathrm{~mm}$ diameter, were drawn on contrast enhanced components in upper, mid, and lower poles of each lymph node and dynamic curves were plotted in a special workstation (Leonardo running workspace-mean curve). In order to determine the most probable metastatic lymph node, dynamic curves of the lymph nodes with the most abnormal contrast morphology (asymmetric cortical thickening, asymmetric contrast enhancement, nodular corticomedullary foci, hilar fat loss, rounding) were plotted, and assessments were based on these curves. Dominant pattern was defined as the pattern predominating in more than $2 / 3$ of all measurements (upper, mid and lower poles of each lymph node).

Even if evaluation was performed in more than one lymph node, dynamic MRI features of the axilla were evaluated by means of average, as axilla was evaluated as a whole during surgery. Main curve types representing graphic data samples obtained are schematized (Figure 1).

\subsection{Benign Cases}

Patients $(n=21)$ in whom no lesions was observed were classified as Breast Imaging Reporting and Data System (BIRADS) category 1. BIRADS category II consisted of patients with fibrocystic changes [adenosis $(\mathrm{n}=7)$, focal hyperplastic unmarked hypoechogenic changes $(\mathrm{n}=9)$ ], those with ductal ectasia $(\mathrm{n}=11)$, those with simple $(\mathrm{n}=$ $29)$ and complicated $(n=7)$ cysts, those with fibroadenoma $(\mathrm{n}=24)$ and those with mastitis [granulomatous mastitis $(\mathrm{n}=1)$, puerperal mastitis $(\mathrm{n}=3)]$.

Of benign cases, 6 patients had unilateral; the remaining 85 patients had bilateral axillary lymph nodes. Thus, 


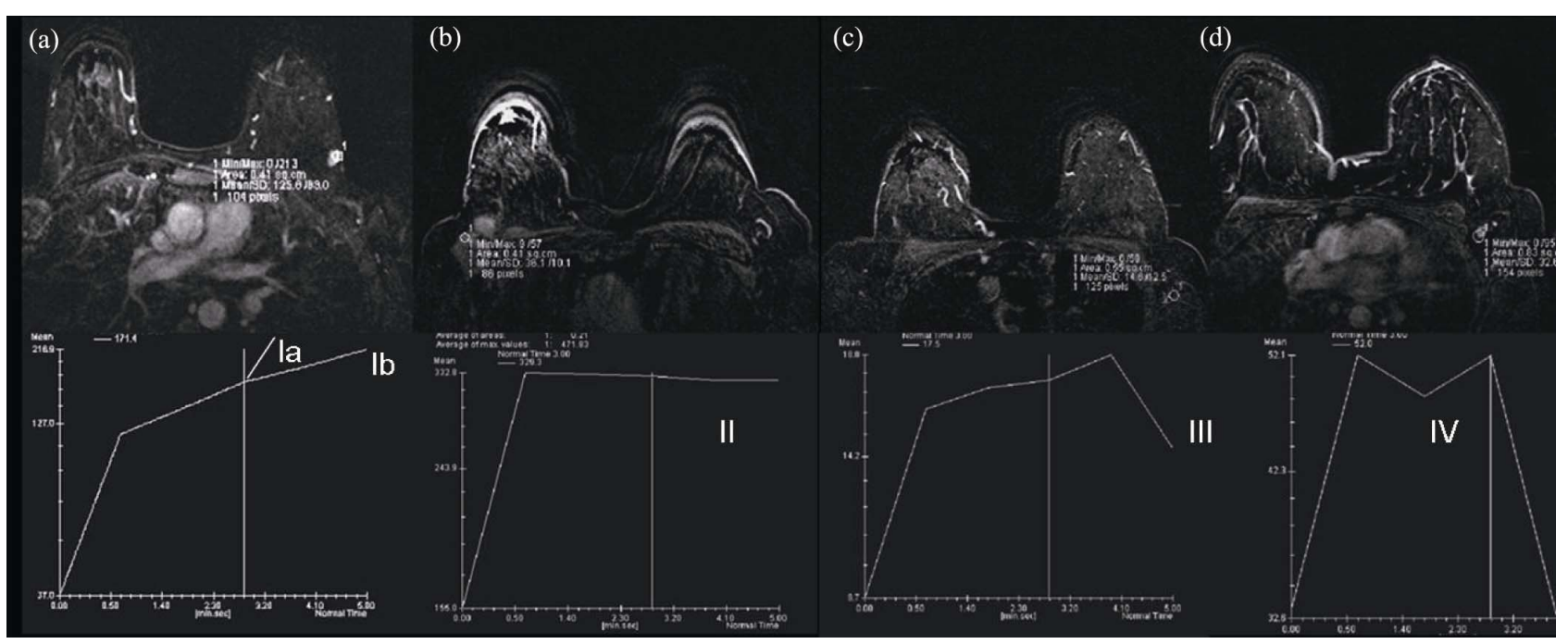

Figure 1. Schematic representation of various types of contrast kinetics. (a) Progressive contrast enhancement in Type Ia (dotted line) and Type Ib patterns; (b) Type II pattern, stable increase of intensity following an initial upstroke, like a plateau; (c) Type III pattern, washout pattern with a fall component following an initial upstroke; (d) Type IV pattern, pattern demonstrating an atypical configurations that may consist of a component from other types but are unpredictable.

ROIs were drawn on bilateral dominant lymph nodes in 85 cases and on unilateral dominant lymph nodes in 6 cases, and curves were plotted.

\subsection{Malignant Cases}

A total of 29 malignant breast lesions (7 diagnosed as lobular carcinoma, 22 as ductal carcinoma) were present. Measurements were performed on the axillary dominant lymph node both at the affected and at the unaffected side in all cases. On histopathologic examination of the affected side in 29 malignant cases, 21 patients were reported to be positive for metastatic involvement, and 8 patients were reported to be negative.

In the present study, 176 (85 bilateral, 6 unilateral) dominant lymph nodes were evaluated in 85 benign cases and 58 lymph nodes consisting of 21 metastatic and 8 reactive lymph nodes (on the ipsilateral side) and 29 benign-reactive lymph nodes (on the contralateral side) were evaluated in 29 malignant cases. Thus, measurements were performed on a total of 234 lymph nodes. Benignity was confirmed based on the 3-year follow-up results and no further histopathologic examination was required (except for one patient with round lymph nodes). Of these patients, 1 patient was evaluated by fine needle aspiration biopsy (FNAB) and the other two were further evaluated by surgical exploration.

In all cases diagnosed with primary breast malignancy, evaluation involved ipsilateral axillary dissection. Lymph nodes on the unaffected side of the cases with malignancy were evaluated based on 3-year follow-up and were reported to be benign.

\subsection{Statistical Analysis}

All study data were summarized in Table 1. Comparisons of groups in terms of age distribution and demographic characteristics were performed by independent samples ttest and Chi-square test, respectively. Chi-square test was also used to assess the relationship between the groups in terms of pattern distribution. All statistical analyses were performed using SPSS Version 15.0.1 (SPSS, Inc., Chicago, IL). Statistical significance was accepted when $\mathrm{p}<$ 0.05 .

\section{Results}

There were no new lesions suspected of malignancy in any of the benign cases (for whom 3-year follow-up data were available) included in the study.

Of 29 patients known to have malignant breast lesions, 21 patients were reported to be positive for axillary lymph node metastasis. No new lesions or other findings suggesting development of malignancy were noted regarding unaffected axilla side of malignant cases in 2- or 3-year follow-up data.

A statistically significant difference was noted between the malignant and benign patients in terms of age, and malignant cases were significantly older $(p<0.05$; independent samples t-test). No statistically significant difference was found between the malignant and benign patients in terms of other demographic characteristics $(p$ $>0.05$; chi-square test).

When signal intensity-time curves were evaluated, in reactive-nonmetastatic lymph nodes Type Ia, Type Ib, 
Table 1. Various signal intensity-time curves of the whole study population.

\begin{tabular}{|c|c|c|c|}
\hline & $\mathbf{n}$ & $\begin{array}{c}\text { Distribution rates of } \\
\text { curve types } \dagger\end{array}$ & $\mathbf{n}$ \\
\hline \multicolumn{4}{|l|}{ Benign cases $(n=91)$} \\
\hline \multicolumn{4}{|l|}{ Bilateral $(n=170)$} \\
\hline Fibrocystic changes & 13 & Type Ia & 1 \\
\hline Ductal ectasia & 10 & Type Ib & 5 \\
\hline Simple cyst & 27 & Type II & 11 \\
\hline Complicated cyst & 7 & Type III & 121 \\
\hline Fibroadenoma & 24 & Type IV & 32 \\
\hline Mastitis & 4 & & \\
\hline \multicolumn{4}{|l|}{ Unilateral $(n=6)$} \\
\hline Fibrocystic changes & & Type Ia & 0 \\
\hline Ductal ectasia & 3 & Type Ib & 1 \\
\hline \multirow[t]{3}{*}{ Simple cyst } & 1 & Type II & 0 \\
\hline & 2 & Type III & 3 \\
\hline & & Type IV & 2 \\
\hline \multicolumn{4}{|l|}{ Malignant Cases $(n=29)$} \\
\hline \multirow{6}{*}{$\begin{array}{c}\text { Tumor side, } \\
\text { metastasis-positive } \\
\text { FNAB } \$ \text { /surgery }\end{array}$} & & Type Ia & 2 \\
\hline & & Type Ib & 3 \\
\hline & 21 & Type II & 12 \\
\hline & & Type III & 2 \\
\hline & & Type IV & 2 \\
\hline & & Type Ia & 0 \\
\hline \multirow{4}{*}{$\begin{array}{c}\text { Tumor side, } \\
\text { metastasis-negative } \\
\text { FNAB } \$ \text { /surgery }\end{array}$} & & Type Ib & 2 \\
\hline & 8 & Type II & 1 \\
\hline & & Type III & 3 \\
\hline & & Type IV & 2 \\
\hline \multirow{5}{*}{ Nontumor side } & & Type Ia & 2 \\
\hline & & Type Ib & 4 \\
\hline & 29 & Type II & 1 \\
\hline & & Type III & 14 \\
\hline & & Type IV & 8 \\
\hline
\end{tabular}

$\dagger$ Type Ia: Progressive straight contrast enhancement, Type Ib: Progressive curved contrast enhancement, Type II: Plateau type contrast enhancement, Type III: Washout type contrast enhancement, Type IV: Nonclassified or atypical graphical contrast enhancement. $¥$ FNAB: Fine needle aspiration biopsy.

Type II, Type III, and Type IV curves were identified in
$1.4 \%, 5.6 \%, 6.2 \%, 66.2 \%$ and $20.6 \%$. In metastatic lymph nodes Type Ia, Type Ib, Type II, Type III and Type IV curves was identified in $9.5 \%, 14.3 \%, 57.2 \%$, $9.5 \%, 9.5 \%$.

Curves in some benign lymph nodes $(\mathrm{n}=7 \%, 3.2 \%)$, which has graphics initially as Type III but continued atypically were also categorized as Type IV. To avoid confounding of the results, a separate category was not constituted for this group. The distribution of signal intensity-time curves for each group is presented in Table 2.

There was no significant difference between malignant and benign cases in terms of the distribution of Type Ia, Type Ib, Type IV curves $(\mathrm{p}=0.12)$. However, a significant difference was noted between the two groups in terms of the distributions of Type II and Type III signalintensity curves $(p<0.01)$. Type II pattern was significantly more frequent in malignant cases and Type III pattern was significantly more frequent in benign cases. The frequency of observing Type II curves was higher with an odds ratio (OR) of 20.5 in malignant cases [95\% Confidence interval (CI), 7.31 - 57.49]. The frequency of observing Type III curves was higher in benign cases with an OR of 0.05 (95\% CI, $0.01-0.23)$. Thus, while Type II pattern is a strong indicator of malignancy, Type III reflects a protective character for benign lesions.

\section{Discussion}

Determination of axillary lymph node involvement in breast cancer is important in terms of treatment protocols, follow-up and prediction of prognosis. Several studies

Table 2. Distribution of various types of signal intensity- time curves among malignant and benign type axillary lymph nodes.

\begin{tabular}{cccc}
\hline Malignant & $\mathbf{n}(\%)$ & Benign & $\mathbf{n}(\%)$ \\
\hline Type Ia & $2(9.52381)$ & Type Ia & $3(1.408451)$ \\
Type Ib & $3(14.2857)$ & Type Ib & $12(5.633803)$ \\
Type II & $12(57.2857)$ & Type II & $13(6.103286)$ \\
Type III & $2(9.52381)$ & Type III & $141(66.19718)$ \\
Type IV & $2(9.52381)$ & Type IV & $44(20.65728)$ \\
Total (n) & 21 & & \\
\hline
\end{tabular}


have reported that physical examination and imaging findings and related sensitivity and specifity findings about the detection of involved axillary lymph node(s) (Table 3). Only imaging with mammography, US, and radionuclide techniques, including positron emission tomography, are currently inadequate or unnecessary; whether or not they are used alone or in combination $[7,37$, 38]. Although providing more effective results, extensive axillary dissection and only sentinel lymph node dissection are cumbersome procedures requiring teamwork and leading to several drawbacks from hospitalization to a wide range of morbidities. On the other hand, in some cases prognosis and the treatment modality may vary based on the degree of lymh node involvement. Patients with $>4$ positive lymph node involvement may benefit from postoperative radiation therapy at a dose of $25-30$ Gy or high-dose chemotherapy. So, it is necessary and important to determine the degree of axillary lymph node involvement [39]. In order to determine the extent of dissection, sentinel lymph node detection and sampling is currently carried out by lymphoscintigraphy which is a difficult and complex procedure $[1,40]$.

Previously, surgical axillary dissection was used as a standard procedure in treatment of breast cancer [2]. However, axillary lymph node metastasis was not present at initial admission in $75 \%$ of early stage cancers, and in $60 \%$ of all breast cancers in general [3] Dissections performed on cases with no axillary metastasis may be associated with lymphedema, limited arm movements, pain during arm movements, and cosmetic problems in addition to unnecessary time and cost loss[2,3]. Recently, detection of the sentinel lymph node with the highest likelihood of being affected and extension of dissection according to the histology of this node is possible by several methods rather than by routine ALND [41]. By this method, however, metastatic involvement of lymph

Table 3. Pivotal studies investigating modalities for the detection of metastatic axillary lymph nodes, and the sensitivity and specificity values as well as major specifications of these modalities.

\begin{tabular}{|c|c|c|c|}
\hline Modality & Sens (\%) & Spec (\%) & Comments or criteria used \\
\hline Clinical palpation & 68 & 67 & Insufficient $[10]$ \\
\hline CT & 50 & 20 & Insufficient [11] \\
\hline Mammography & 40 & 37 & $\begin{array}{l}\text { Insufficient: Sensitive only in the presence of microcalcifications (3\%) in the lymph node } \\
\text { [12] }\end{array}$ \\
\hline US-Size & $44-87$ & $55-97$ & Insufficient. High sensitivity only in large lymph nodes that are $>3 \mathrm{~cm}$ [13] \\
\hline \multirow[t]{2}{*}{ US-Morphology } & $26-76$ & $88-98$ & Loss of hilar echo, rounding, hypoechogenicity, eccentric cortical thickening [13] \\
\hline & & & $\begin{array}{l}\text { CDUS: Peripheral weighted, vascularization by cortical penetrating vessels, nonuniform } \\
\text { vascularity }[14,15]\end{array}$ \\
\hline \multirow{3}{*}{$\begin{array}{l}\text { CDUS, PDUS, } \\
\text { spectral indices, } \\
\text { echo-enhanced US } \\
\text { Sonoelastography }\end{array}$} & & & PDUS: increased sensitivity to low flow rate $[16,17]$ \\
\hline & 77 & 28 & $\begin{array}{l}\text { Doppler waveform: The resistive index, pulsatility index, and peak systolic velocities in this } \\
\text { population overlapped between benign and malignant lymphadenopathy [18] }\end{array}$ \\
\hline & & & $\begin{array}{l}\text { Microbubble enhanced Doppler US: area under curve is } 0.77 \text {., it is expensive and not } \\
\text { widely used (19), Sonoelastography is a new but promising technique [20] }\end{array}$ \\
\hline $\begin{array}{l}\text { US (combined with } \\
\text { clinical findings) }\end{array}$ & 87 & 74 & Combination of gray scale US with Doppler US findings [21) \\
\hline MRI (conventional) & 55 & 81 & $\begin{array}{l}\text { Primary tumor can be assessed directly and axillary lymph node characteristics can be as- } \\
\text { sessed indirectly by dynamic evaluation [22-26] }\end{array}$ \\
\hline MRI - spectroscopy & 82 & 100 & High sensitivity/specificity except for choline negative in-situ cancers [27] \\
\hline MRI (USPIO) & $80-90$ & $90-100$ & Nanotechnology product, high cost and low availability [22] \\
\hline PET imaging & 96 & 100 & $\begin{array}{l}\text { Radiation exposure, high cost, cannot be used for screening in every patient. Other radionu- } \\
\text { clide techniques are less sensitive than PET imaging }[1,28,29]\end{array}$ \\
\hline $\begin{array}{l}\text { Sonography guided } \\
\text { FNAB }\end{array}$ & $31-63$ & $95-100$ & $\begin{array}{l}\text { Negative biopsy results do not exclude axillary lymph node metastasis }[30,31] \\
\text { Sentinel lymph node biopsy: a cumbersome method, radiation exposure occurs, although be- } \\
\text { ing an invasive method false negativity rate is relatively high [32-36] }\end{array}$ \\
\hline
\end{tabular}


nodes other than detected sentinel lymph nodes has been reported to reach up to $15 \%$ [42]. This false negativity rate is high for such an invasive and complicated procedure, which requires teamwork and is associated with radiation exposure of both the patient and the physician in some versions [42].

Actually, axillary lymph nodes may be palpated initially during routine clinical examination. However, palpation which is routinely used in physical examination has a low sensitivity and specificity for detecting axillary lymphadenopathy [40]. Other methods including mammography, computed tomography (CT), also fails to detect deep axillary lymph nodes $[11,40]$. Sensitivity of this US is also not very high and it has been used in combination with color doppler US (CDUS) and power doppler US (PDUS) [21].

Lymphoscintigraphy, a method that is used to detect the sentinel lymph nodes, has a relatively high sensitivity (95\%); however, non-repeatability and low sensitivity for deeply located axillary and internal mammary chain lymph nodes continue to be a disadvantage of this technique [7]. It is not possible for now to use new molecular methods like "breast cancer-specific monoclonal antibodies in the staging of the axilla" to overcome disadveantages because of being in an experimental stage [43, 44]. Also new nanotechnologic MRI contrast agents (which alleged to reveal the micrometastases, like USPIO) has not gained widespread use because of high costs [22].

Despite all of the ongoing research for an ideal method, the assessment of axillary lymph nodes conveys important prognostic information that is needed to guide stageadapted therapy for breast cancer. As the number of women who are diagnosed with early-stage breast cancer increases, an increasing number of axillary lymph node dissections will yield healthy lymphatic tissue. In order to minimize these unnecessary interventions, we investigated the use of dynamic breast-axillary MRI. We aimed to increase the sensitivity of data acquired by already performed dynamic MRI. In addition to being noninvasive, the main difference of our technique from its previous counterparts is that it combines MRI images, which can visualize all of the potential axillary lymph nodes simultaneously, with dynamic evaluation, which can also provide functional information.

In previous dynamic breast MRI studies, evaluations have focused on primary breast masses and all measurements (types of signal intensity-time curves) and their significance have been directed towards this primary mass [45]. The assessment of the type of signal intensitytime curves (i.e., kinetic curves) by categorizing the washout pattern of a gadolinium contrast agent is the most widely used form of DCE-MRI analysis in breast cancers. These curves are classified as follows: 1) Type I, persistently enhancing (progressive), which is suggestive of benignity; 2) Type II, plateau type, which is suggestive of possible malignancy; and 3) Type III, washout type, which is strongly suggestive of malignancy [45].

Furthermore, in contrast to focusing only on primary lesion located in breast tissue, we obtained also signal intensity-time curves in axillary lymph nodes without performing any additional procedure in patients of whom the dynamic MRI evaluations were already available. We investigated whether the contrast enhancement observed in these dynamic series will be effective in differential diagnosis. It is well recognized that most primary breast cancers show rapid enhancement and rapid washout (Type III curve pattern) on MRI following gadoliniumbased contrast agents [25]. We aimed to investigate whether or not a similar kinetic form can be used for axillary measurements, as well. Literature review conducted for this study revealed a limited number of studies invesitagting dynamic MRI together with axillary lymph nodes [15]. The aim of one of these studies was to determine and compare the response (such as size, number, contrast enhancement level) of axillary lymph nodes to a special chemotherapeutic treatment (trastuzumab) as well as primary lesion kinetics by MRI [15]. Rather than contrast kinetics of axillary lymph nodes in dynamic series, different from the study which concluded that "HER2/ neu-positive patients with a complete clinical response on DCE-MRI are highly unlikely to benefit from an axillary lymph node dissection", we have performed the measurements in the dominant-sentinel lymph node [15]. In another study, association between angiogenetic kinetics of the tumor and the presence of axillary lymph node was investigated using contrast dynamics of primary mass and no relationship was noted between kinetic characteristics and axillary lymph node metastasis [15]. In that particular study, actual kinetic measurements were performed for the primary mass. However, although dynamic contrast technique was used and simultaneous axilla examination was performed in some of these studies, a single feature (signal intensity) was investigated as an enhancement characteristic rather than the time-signal intensity curves, and graphical data were not produced [16]. We have not focused on signal intensity in our study and information regarding the metastatic process, which alters the lymph node anatomy and quality of the vascular bed and consequently changes contrast kinetics, was obtained indirectly from graphical data. In the present study, however, lymph nodes with dominant morphological or kinetic were marked without taking into consideration any other breast lesion into account (no matter if the primary pathology is benign or malignant). Our main goal was to analyze the 
kinetic characteristics of the axillary dominant lymph node which may have been presumed to be affected from the primary breast lesion at first.

In two of previous related studies, a similar method that used in the present study was also utilized to detect axillary lymph node metastases of breast cancer $[9,46]$. There were technical differences in the calculation of maximum contrast enhancement ratios among these studies in which the dynamic contrast enhancement ratio, sizes and also the morphology of the related axillary lymph nodes were recorded. However, in general when using a signal intensity increase in the lymph nodes of $>100 \%$ during the first postcontrast image as a threshold for malignancy, 57 of 65 patients were correctly classified (sensitivity $83 \%$, specificity $90 \%$, accuracy $88 \%$ ) [9]. These relatively significant sensitivity rates were not improved when lymph node size and morphology were used as additional criteria [9]. We did not take this data and other morphological criteria into account for other reasons or include them in statistical analysis. We believed that we reached our goal since we achieved interestingly high sensitivity and specificity values without performing complicated calculations and leading to confusion.

Our findings were objectively confirmed by current histopathologic findings. Normally, a lymph node is a small bean-shaped structure located in stations such as axilla and contains phagocytic cells, which acts as filters for particulate matter and micro-organisms, and the antigen, which is presented to the immune system. A normal node consists of three components; lymphatic sinuses, blood vessels, and parenchyma (cortex, paracortex, medulla). There is also an outer fibrous capsule. While normally the arteries and veins in a lypmh node connect with a short capillary network; with the metastasis the blood circulates in a slower fashion and needs to stay longer in that enlarged vascular bed which is expanded due to the proliferation of malignant cells and angiogenesis. In contrast to the rapid washout pattern observed in normal lymph nodes, contrast enhancement observed as a plateau pattern in metastatic lymph nodes can be explained by this phenomenon. There are also several studies in which perfusion changes caused by this organization have been investigated [17,47]. In Figure 2, comparative hystopathological stained sections from normal and metastatic lymph nodes are presented.

The present study had somel limitations. The most important limitations were the partial appearance of axilla in some of the image fields and the presence artifactual distorsions due to respiratory movements or intensive venous contrast accumulation on the side of contrast administration. In order to overcome these limitations, we believe that it would be helpful to train the patients so that they continued to breathe at a more tidal level. And tothe clinical examination the normal side of the arm must be used for contrast administration. Another limitation was that only those cases with routine ALND results were included in the study; and the dissected lymph node was accepted as the measured dominant lymph node at MRI. But, as we know, there are no literature data about to marking the axillary lymph node(s) under the guidance

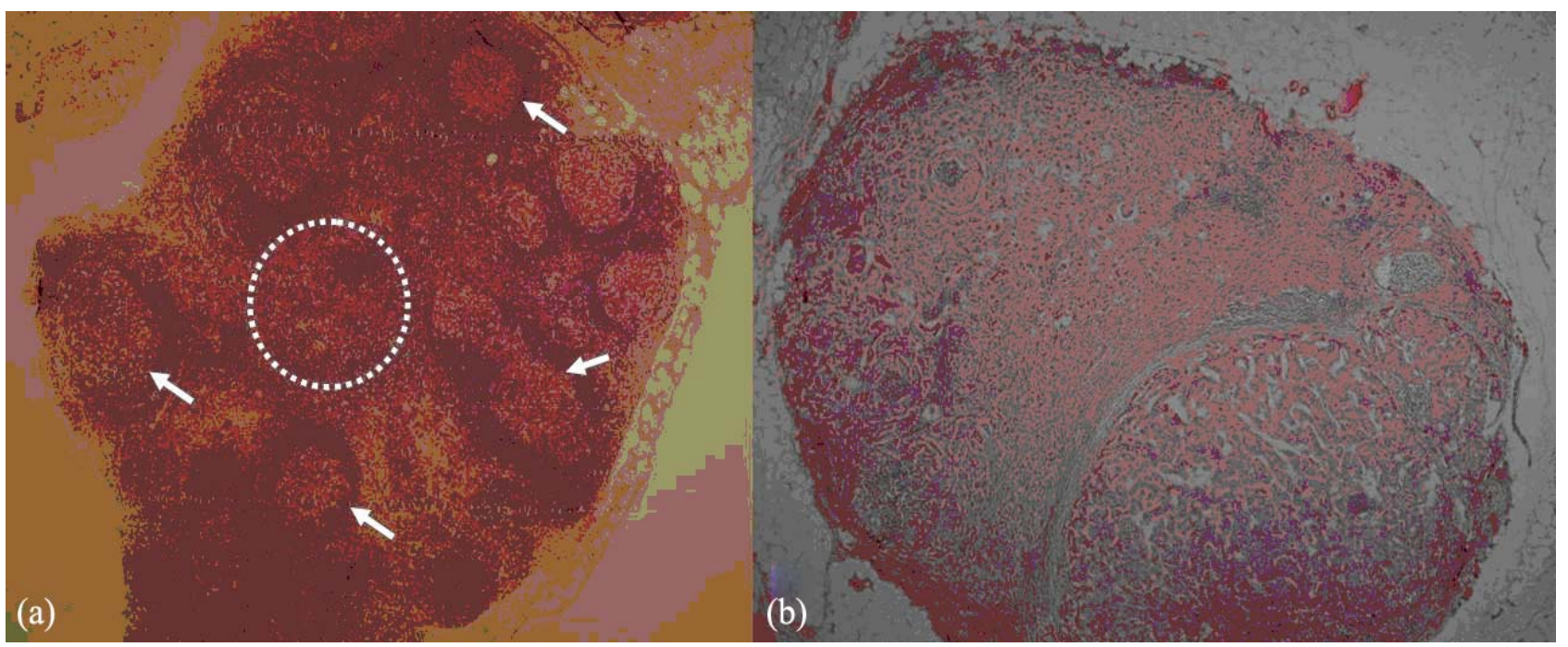

Figure 2. Etiopathogenetic theory which may explain the difference in contrast kinetics in dynamic series. (a) In a normal lymph node, cortical follicles (arrows) are distributed throughout the lymph node. The outer cortex is full of lymph follicles (B lymphocytes at the center, $T$ lymphocytes at the periphery); inner medulla (circle) is full of lymphocytes, macrophages, plasma cells (activated B cells). (b) In a metastatic lymph node, normal microanatomy disappears and metastatic cells are distributed in a disorganized fashion. $\mathrm{H} \& \mathrm{E}$ stain, $\times 10$. 
of MRI. We also have controlled the descrition of the location of dissected lymph nodes from the surgery reports and have seen that these locations were the same as at the MRI reports.

In conclusion, the method used in the present study was a functional assessment measuring the contrast dynamics formed within the microenvironment of the lymph node. In cases with known primary breast mass, kinetic characteristics of axillary lymph nodes can be obtained from signal intensity-time curves by using DCE breast MRI images obtained by extending of FOV towards the axillary fossa. Contrast enhancement kinetics Type Ia, Ib, II (57.2\%) are more common in malignant axillary lymph nodes; Type III (66.2\%) and Type IV signal intensity curves are more common in benign lymph nodes. The mechanism underlying these contrast kinetics may be associated with metastasis related increased vascularity in the lymph node due to prolongation of the distance traversed by the contrast agent and the time the contrast agent remained within the lymph node without being rapidly washed-out. We believe that this method will improve the sensitivity of routine DCE-Breast MRI examination in determining the axillary lymph node status.

\section{REFERENCES}

[1] L. P. Adler, J. P. Crowe, N. K. al-Kaisi and J. L. Sunshine, "Evaluation of Breast Masses and Axillary Lymph Nodes with [F-18] 2-Deoxy-2-fluoro-D-glucose PET," Radiology, Vol. 187, 1993, pp. 743-750.

[2] U. Veronesi, G. Paganelli, G. Viale G, et al., "Sentinel Lymph Node Biopsy and Axillary Dissection in Breast Cancer: Results in a Large Series," Journal of National Cancer Institute, Vol. 91, No. 4, 1999, pp. 368-373. doi:10.1093/jnci/91.4.368

[3] B. Cady, "The Need to Reexamine Axillary Lymph Node Dissection in Invasive Breast Cancer," Cancer, Vol. 73, 1994, pp. 505-508.

[4] W. K. Ruffin, A. Stacey-Clear, J. Younger and H. C. Hoover, "Rationale for Routine Axillary Dissection in Carcinoma of the Breast," Journal of the American College of Surgeons, Vol. 180, 1995, pp. 245-251.

[5] A. Recht and M. J. Houlihan, "Axillary Lymph Nodes and Breast Cancer: A Review," Cancer, Vol. 76, No. 9, 1995, pp. 1491-1512.

[6] B. Fowble, R. Gray, K. Gilchrist, et al., "Identification of a Subgroup of Patients with Breast Cancer and Histologically Positive Axillary Nodes Receiving Adjuvant Chemotherapy Who may Benefit from Postoperative Radiotherapy," Journal of Clinical Oncology, Vol. 6, 1988, pp. 1107-1117.

[7] R. G. McLean and G. N. Ege, "Prognostic Value of Axillary Lymphoscintigraphy in Breast Carcinoma Patients,"
Journal of Nuclear Medicine, Vol. 27, 1986, pp. 11161124.

[8] S. E. Harms, D. P. Flamig, K. L. Hesley, et al., "MR Imaging of the Breast with Rotating Delivery of Excitation off Resonance: Clinical Experience with Pathologic Correlation," Radiology, Vol. 187, 1993, pp. 493-501.

[9] A. D. Murray, R. T. Staff, T. W. Redpath, et al., "Dynamic Contrast Enhanced MRI of the Axilla in Women with Breast Cancer: Comparison with Pathology of Excised Nodes," The British Journal of Radiology, Vol. 75, 2002, pp. 220-228.

[10] B. Cady, "Dilemmas in Breast Disease," The Breast Journal, Vol. 1, No. 2, 1995, pp. 121-124. doi:10.1111/j.1524-4741.1995.tb00230.x

[11] D. E. March, R. J. Wechsler, A. F. Kurtz, A. L. Rosenberg and L. Needleman, "CT-Pathologic Correlation of Axillary Lymph Nodes in Breast Carcinoma," Journal of Computer Assisted Tomography, Vol. 15, No. 3, 1991, pp. 440-444. doi:10.1097/00004728-199105000-00017

[12] L. Kalisher, A. M. Chu and R. G. Peyster, "Clinicopathological Correlation of Xeroradiography in Determining Involvement of Metastatic Axillary Nodes in Female Breast Cancer," Radiology, Vol. 121, 1976, pp. 333-335.

[13] S. Alvarez, E. Añorbe, P. Alcorta, F. López, I. Alonso and J. Cortés, "Role of Sonography in the Diagnosis of Axillary Lymph Node Metastases in Breast Cancer: A Systematic Review," American Journal of Roentgenology, Vol. 186, 2006, pp. 1342-1348.

[14] W. T. Yang, C. Metreweli, P. K. Lam and J. Chang, "Benign and Malignant Breast Masses and Axillary Nodes: Evaluation with Echo-Enhanced Color Power Doppler US," Radiology, Vol. 220, 2001, pp. 795-802. doi:10.1148/radiol.2203001545

[15] W. T. Yang, J. Chang and C. Metreweli, "Patients with Breast Cancer: Differences in Color Doppler Flow and Gray-Scale US Features of Benign and Malignant Axillary Lymph Nodes," Radiology, Vol. 215, 2000, pp. 568573.

[16] H. J. Steinkamp, U. K. Teichgräber, M. Mueffelmann, N. Hosten and P. Kenzel, "Felix. Differential Diagnosis of Lymph Node Lesions. A Semiquantitative Approach with Power Doppler Sonography," Investigative Radiology, Vol. 34 , No. 8, pp. 509-515. doi:10.1097/00004424-199908000-00003

[17] H. J. Steinkamp, C. Wissgott, J. Rademaker and R. Felix, "Current Status of Power Doppler and Color Doppler Sonography in the Differential Diagnosis of Lymph Node Lesions," European Radiology, Vol. 12, 2002, pp. 1785 1793.

[18] M. Y. Choi, J. W. Lee and K. J. Jang, "Distinction between Benign and Malignant Causes of Cervical, Axillary, and Inguinal Lymphadenopathy: Value of Doppler Spectral Wave-Form Analysis," American Journal of Roentgenology, Vol. 165, 1995, pp. 981-984.

[19] A. R. Sever, P. Mills, S. E. Jones, K. Cox, J. Weeks, D. Fish and P. A. Jones, "Preoperative Sentinel Node Identi- 
fication with Ultrasound Using Microbubbles in Patients with Breast Cancer," American Journal of Roentgenology, Vol. 196, No. 2, 2011, pp. 251-256.

[20] J. J. Choi, B. J. Kang, S. H. Kim, et al., "Role of Sonographic Elastography in the Differential Diagnosis of Axillary Lymph Nodes in Breast Cancer," Journal of Ultrasound in Medicine, Vol. 30, 2011, pp. 429-436.

[21] P. Vassallo, G. Edel, N. Roos, A. Naguib and P. E. Peters, "In-Vitro High-Resolution Ultrasonography of Benign and Malignant Lymph Nodes: A Sonographic-Histopathologic Correlation," Investigative Radiology, Vol. 28, No. 8, 1993, pp. 698-705. doi:10.1097/00004424-199308000-00009

[22] M. Memarsadeghi, C. C. Riedl, A. Kaneider, A. Galid, M. Rudas, W. Matzek and T. H. Helbich, "Axillary Lymph Node Metastases in Patients with Breast Carcinomas: Assessment with Nonenhanced versus USPIO-Enhanced MR Imaging," Radiology, Vol. 241, 2006, pp. 367-377.

[23] E. R. Horak, R. Leek, N. Klenk, et al., "Angiogenesis, Assessed by Platelet/Endothelial Cell Adhesion Molecule Antibodies, as Indicator of Node Metastases and Survival in Breast Cancer," Lancet, Vol. 340, 1992, pp. 1120-1124.

[24] N. Weidner, P. Semplej, W. R. Welch and J. Folkman, "Tumor Angiogenesis and Metastasis: Correlation in Invasive Breast Carcinoma," The New England Journal of Medicine, Vol. 324, 1991, pp. 1-8. doi:10.1056/NEJM199101033240101

[25] S. E. Harms, D. P. Flamig, K. L. Hesley, et al., "MR Imaging of the Breast with Rotating Delivery of Excitation off Resonance: Clinical Experience with Pathologic Correlation," Radiology, Vol. 187, 1993, pp. 493-501.

[26] E. Furman-Haran, R. Margalit, D. Grobgeld and H. Dcgani, "Dynamic Contrast-Enhanced Magnetic Resonance Imaging Reveals Stress-Induced Angiogenesis in MCF7 Human Breast Tumors," The Proceedings of the National Academy of Sciences, Vol. 93, No. 13, 1996, pp. 6247- 6251. doi:10.1073/pnas.93.13.6247

[27] D. K. Yeung, W. T. Yang and G. M. Tse, "Breast Cancer: In Vivo Proton MR Spectroscopy in the Characterization of Histopathologic Subtypes and Preliminary Observations in Axillary Node Metastases," Radiology, Vol. 225, 2002, pp. 190-197. doi:10.1148/radiol.2243011519

[28] R. L. Wahl, R. L. Cody, G. D. Hutchins and E. E. Mudgett, "Primary and Metastatic Breast Carcinoma: Initial Clinical Evaluation with Pet with the Radiolabeled Glucose Analogue 2-[F-18]-Fluoro-2-deoxy-O-glucose," Radiology, Vol. 179, 1991, pp. 765-770.

[29] H. Minn and I. Soini, "[18F]Fluorodeoxyglucose Scintigraphy in Diagnosis and Follow up of Treatment in Advanced Breast Cancer," European Journal of Nuclear Medicine, Vol. 15, No. 2, 1989, pp. 61-66. doi:10.1007/BF00702620

[30] M. R. Keshtgar and M. Baum, "Axillary Dissection over the Years: Where to from Here?" World Journal of Surgery, Vol. 25, No. 6, pp. 761-766. doi:10.1007/s00268-001-0002-y

[31] B. J. Grube and A. E. Giuliano, "The Current Role of
Sentinel Node Biopsy in the Treatment of Breast Cancer," Advences in Surgery, Vol. 38, 2004, pp. 121-166.

[32] J. S. Jeruss, D. J. Winchester, S. F. Sener, et al., "Axillary Recurrence after Sentinel Node Biopsy," Annals of Surgical Oncology, Vol. 12, No. 1, 2005, pp. 34-40. doi:10.1007/s10434-004-1164-2

[33] M. L. Smidt, C. M. Janssen, D. M. Kuster, E. D. Bruggink and L. J. Strobbe, "Axillary Recurrence after a Negative Sentinel Node Biopsy for Breast Cancer: Incidence and Clinical Significance," Annals of Surgical Oncology, Vol. 12, 2005, pp. 29-33.

[34] S. Krishnamurthy, N. Sneige, D. G. Bedi, et al., "Role of Ultrasound-Guided Fine-Needle Aspiration of Indeterminate and Suspicious Axillary Lymph Nodes in the Initial Staging of Breast Carcinoma," Cancer, Vol. 95, No. 5, 2002, pp. 982-988. doi:10.1002/cncr.10786

[35] A. Sapino, P. Cassoni, F. Zanon, et al., "Ultrasonographically-Guided Fine-Needle Aspiration of Axillary Lymph Nodes: Role in Breast Cancer Management," British Journal of Cancer, Vol. 88, 2003, pp. 702-706. doi:10.1038/sj.bjc. 6600744

[36] J. U. Oruwari, M. A. Chung, S. Koelliker, M. M. Steinhoff and B. Cady, "Axillary Staging Using Ultrasoundguided Fine Needle Aspiration Biopsy in Locally Advanced Breast Cancer," The American Journal of Surgery, Vol. 184, 2002, pp. 307-309.

[37] M. Pamilo, M. Soiva and E. M. Lavast, "Real-Time Ultrasound, Axillary Mammography, and Dynamical Examination in the Detection of Axillary Lymph Node Metastases in Breast Cancer Patients," Journal of Ultrasound in Medicine, Vol. 8, 1989, pp. 115-120.

[38] J. J. Tjandra, N. P. M. Sacks, C. H. Thompson, et al., "The Detection of Axillary Lymph Node Metastases from Breast Cancer by Radiolabelled Monoclonal Antibodies: A Prospective Study," British Journal of Cancer, Vol. 59, 1989, pp. 296-302. doi:10.1038/bjc.1989.61

[39] S. S. Jeffrey, S. B. Jones and K. Smith, "Controversies in Sentinel Lymph Node Biopsy for Breast Cancer," Cancer Biotherapy and Radiopharmaceuticals, Vol. 15, No. 3, 2000, pp. 223-233. doi:10.1089/108497800414310

[40] D. E. March, R. J. Wechsler, A. F. Kurtz, A. L. Rosenberg and L. Needleman, "CT-Pathologic Correlation of Axillary Lymph Nodes in Breast Carcinoma," Journal of Computer Assisted Tomography, Vol. 15, No. 3, 1991, pp. 440-444. doi:10.1097/00004728-199105000-00017

[41] M. J. Silverstein, E. D. Gierson, J. R. Waisman, G. M. Senofsky, W. J. Colbum and P. Gamagani, “Axillary Lymph Node Dissection for Tla Breast Carcinoma," Cancer, Vol. 73, 1994, pp. 664-667.

[42] M. Fraile, M. Rull, F. J. Julián, et al., "Sentinel Node Biopsy as a Practical Alternative to Axillary Lymph Node Dissection in Breast Cancer Patients: An Approach to Its Validity," Annals of Oncology, Vol. 11, 2000, pp. 701705.

[43] H. Fujii, K. Enomoto, T. Ikeda, et al., "Lymph Node Metastasis from Breast Cancer Diagnosed by F-18 FDG 
Whole-Body PET," Breast Cancer, Vol. 7, No. 2, 2000, pp. 165-168. doi:10.1007/BF02967451

[44] N. Y. Tse, C. K. Hoh, R. A. Hawkins, et al., "The Application of Positron Emission Tomographic Imaging with Fluorodeoxyglucose to the Evaluation of Breast Disease," Annals of Surgery, Vol. 216, No. 1, 1992, pp. 27-34.

[45] R. H. El Khouli, K. J. Macura, M. A. Jacobs, et al., "Dynamic Contrast-Enhanced MRI of the Breast: Quantitative Method for Kinetic Curve Type Assessment," American Journal of Roentgenology, Vol. 193, No. 4, 2009, pp. 295-300. doi:10.2214/AJR.09.2483
[46] P. Schrenk, A. Shamiyeh and W. Wayand, "Sentinel Lymph-Node Biopsy Compared to Axillary Lymph-Node Dissection for Axillary Staging in Breast Cancer Patients," European Journal of Surgical Oncology, Vol. 27, 2001, pp. 378-382. doi:10.1053/ejso.2001.1139

[47] W. T. Yang, G. M. Tse, P. K. Lam, C. Metreweli and J. Chang, "Correlation between Color Power Doppler Sonographic Measurement of Breast Tumor Vasculature and Immunohistochemical Analysis of Microvessel Density for the Quantitation of Angiogenesis," Journal of Ultrasound in Medicine, Vol. 21, 2002, pp. 1227-1235. 